\title{
Sunitinib im klinischen Alltag: Das Expanded-Access- Programm beim metastasierten Nierenzellkarzinom
}

\author{
Norbert Marschner \\ Praxis für interdisziplinäre Onkologie und Hämatologie, Freiburg i.Br.
}

\section{Schlüsselwörter}

Metastasiertes Nierenzellkarzinom · Sunitinib .

Expanded-Access-Programm · Klinischer Alltag ·

Erstlinientherapie $\cdot$ Zweitlinientherapie

\section{Zusammenfassung}

Der präferierte Einsatz von Sunitinib in der Erstlinientherapie des metastasierten Nierenzellkarzinoms (mRCC) beruht auf den Daten einer Phase-III-Studie, in der mit dem Multi-Kinase-Inhibitor eine Verdopplung des progressionsfreien Überlebens (PFS) gegenüber Interferon-alpha (IFN- $\alpha$ ) erreicht werden konnte. Die Daten aus klinischen Studien werden nun ergänzt und gestützt durch umfassende Erfahrungen aus dem Praxisalltag. Innerhalb des Sunitinib-Expanded-Access-Programms mit mehr als 4500 Teilnehmern wurde Sunitinib einem erweiterten Patientenkreis zugänglich gemacht. Daher konnten auch Patienten eingeschlossen werden, die aufgrund ihrer länderspezifischen Zulassungssituation bisher keinen Zugang zu einer Therapie mit Sunitinib hatten oder die an der Zulassungsstudie aufgrund von Ausschlusskriterien nicht teilnehmen konnten. Das Kollektiv umfasste auch Patienten mit ungünstiger Prognose, beispielsweise aufgrund von Hirnmetastasen, nichtklarzelligem RCC oder einem ECOG-Performance-Status $\geq 2$. Im Vordergrund standen Fragen zur Sicherheit und Langzeitverträglichkeit von Sunitinib im klinischen Alltag. Hinsichtlich der Verträglichkeit unterschieden sich die Subgruppen nicht von der Gesamtpopulation. Es traten keine neuen oder kumulativen Toxizitäten auf. Darüber hinaus zeigte sich, dass auch die prognostisch ungünstigen Subgruppen von einer Therapie mit Sunitinib profitieren. In der Zusammenschau von Phase-III-Daten und den konsistenten Ergebnissen des internationalen Sunitinib-ExpandedAccess-Programms verfügt Sunitinib über eine breite Evidenzbasis für den Einsatz in allen Patientenpopulationen.

Sunitinib als ein Referenzstandard beim metastasierten Nierenzellkarzinom

Sunitinib ist ein Referenzstandard beim fortgeschrittenen/metastasierten Nierenzellkarzinom (mRCC). In der Phase-III-Studie zur Erstlinientherapie zeigten Patienten im Sunitinib-Arm ein um 6 Mo-

\section{Key Words}

Metastatic renal cell carcinoma - Sunitinib .

Expanded access program - Clinical practice · First-line therapy . Second-line therapy

\section{Summary}

Sunitinib in Clinical Practice: the Expanded Access Program for Metastatic Renal Cell Carcinoma

The preferential application of Sunitinib in the first-line therapy of metastatic renal cell carcinoma ( $\mathrm{mRCC}$ ) is based on data of a phase III study documenting a doubling of progression-free survival (PFS) with the multi-kinase inhibitor compared to Interferon-alpha. Data from clinical studies were then supplemented and supported by comprehensive experiences in everyday practice. Sunitinib was offered to a large group of patients in an expanded access program with more than 4,500 participants. Patients with no access to Sunitinib treatment due to their country-specific approval situation or patients unable to participate in the approval study on the basis of exclusion criteria could thus be included. The cohorte also encompassed patients with an unfavorable prognosis due to metastases of the brain, non-clear cell RCC, or an ECOG performance status $\geq 2$. Priority was given to questions regarding the safety and long-term tolerability of Sunitinib in clinical practice. Regarding tolerability, these subgroups in the expanded access program did not differ from the total population. New or cumulative toxicities did not occur. In addition, even subgroups with an unfavorable prognosis were able to profit from treatment with Sunitinib. A synopsis of phase III data and the results of the expanded access program confirm the evidence for Sunitinib in all patient populations.

\section{KARGER}

Fax +497614520714

Information@Karger.de

www.karger.com (c) 2010 S. Karger GmbH, Freiburg

Accessible online at:

www.karger.com/onk nate längeres progressionsfreies Überleben (PFS) im Vergleich zu Interferon-alpha (IFN- $\alpha$ ) (median 11,2 vs. 5,1 Monate; $\mathrm{p}<0,001)$. Als sekundärer Endpunkt wurde eine Vervierfachung der objektiven Ansprechrate auf 47 versus $12 \%(\mathrm{p}<0,001)$ beobachtet [1]. Zudem erreichten die Patienten unter Sunitinib ein medianes Gesamtüberleben von 26,4 Monaten. Unter IFN- $\alpha$ betrug das Gesamtüberleben 


\begin{tabular}{|c|c|c|c|c|c|}
\hline \multicolumn{6}{|c|}{$\begin{array}{l}\text { Effektivität im Sunitinib-Expanded-Access-Programm: } \\
\text { Gesamtpopulation und Subgruppen }\end{array}$} \\
\hline & Gesamt & $\geq 65$ Jahre & $\begin{array}{l}\text { nicht- } \\
\text { klarzellige } \\
\text { Histologie }\end{array}$ & $\begin{array}{c}\text { Hirn- } \\
\text { metastasen }\end{array}$ & $\begin{array}{l}\text { ECOG } \\
\text { PS } \geq 2\end{array}$ \\
\hline Patientenzahl, n & 4.349 & 1.056 & 437 & 213 & 319 \\
\hline $\begin{array}{l}\text { PFS } \\
\text { (Monate) }\end{array}$ & 10,9 & $\left(\begin{array}{c}11,3 \\
(10,7-12,3)\end{array}\right.$ & $\left(6,3^{7,8}-8,3\right)$ & $\begin{array}{c}5,6 \\
(5,2-6,1)\end{array}$ & $(4,2-5,5)$ \\
\hline $\begin{array}{l}\text { Klinischer Nutzen } \\
\text { (CR + PR + SD) }\end{array}$ & $\begin{array}{c}76 \% \\
(n=3.464)\end{array}$ & $77 \%$ & $68 \%$ & $64 \%$ & $61 \%$ \\
\hline
\end{tabular}

Abb. 1. Das EAP zeigte die gute Wirksamkeit von Sunitinib auch bei prognostisch ungünstigsten Subgruppen, wie bei mRCC-Patienten mit Hirnmetastasen, nichtklarzelliger Tumorhistologie, schlechtem Allgemeinzustand (ECOG-PS $\geq 2$ ) oder einem Alter $\geq 65$ Jahre.

21,8 Monate ( $\mathrm{p}=0,051)$ [2]. Für die überwiegende Mehrheit der Patienten ist Sunitinib derzeit eine First-line-Therapie der ersten Wahl, zumal auch die Wirksamkeit von Folgetherapien wie beispielsweise Sorafenib belegt werden konnte [3-5].

\section{Widerspiegelung des klinischen Alltags}

Die Evidenzbasis der Phase-III-Studie wird durch Erfahrungen aus dem Praxisalltag auf Basis eines großen Expanded-Access-Programms (EAP) mit mehr als 4500 Patienten ergänzt [6]. Ziel des EAP war es, Sunitinib einem erweiterten Patientenkreis zukommen zu lassen. Daher konnten auch Patienten eingeschlossen werden, die aufgrund ihrer länderspezifischen Zulassungssituation bisher keinen Zugang zu einer Therapie mit Sunitinib hatten oder die an der Zulassungsstudie nicht teilnehmen konnten. Die Einschlusskriterien waren deutlich weiter gefasst, sodass auch Patienten mit ungünstiger Prognose, beispielsweise aufgrund von Hirnmetastasen, nichtklarzelligem RCC oder einem ECOG-Performance-Status(PS) $\geq 2$, aufgenommen wurden.

Im Vordergrund standen dabei Fragen zur Sicherheit und Langzeitverträglichkeit von Sunitinib im klinischen Alltag. Gleichzeitig diente das EAP der Gewinnung von Informationen über die Wirksamkeit in dieser heterogenen Patientengruppe, die sowohl Erstlinien- als auch Zweitlinienpatienten einschloss und damit den klinischen Alltag stärker widerspiegelt als eine Phase-III-Studie.

Sunitinib auch bei schwierig zu behandelnden Subgruppen effektiv und verträglich

Die Ergebnisse des EAP weisen in die gleiche Richtung wie die Phase-III-Daten. So betrug das PFS in der Gesamtpopulation 10,9 Monate (95\% CI 10,3-11,2) und in den Subgruppen 5,1-11,3 Monate. Die beobachteten Nebenwirkungen waren in der Regel leicht bis moderat. Neue oder kumulative Toxizitäten traten nicht auf. Besonders bemerkenswert ist, dass auch Patienten mit ungünstiger Prognose aufgrund von Hirnmetastasen oder schlechtem Allgemeinzustand von Sunitinib profitieren konnten. Diese Subgruppen unterschieden sich auch hinsichtlich der Verträglichkeit von Sunitinib nicht von der Gesamtpopulation.

Die objektive Ansprechrate lag bei $12 \%$ für Patienten mit Hirnmetastasen, $9 \%$ für Patienten mit ECOG-PS $\geq 2$, 9\% für Patienten mit nichtklarzelliger Tumorhistologie und $17 \%$ für Patienten $\geq 65$ Jahre. Der klinische Nutzen betrug in der Gesamtpopulation $76 \%$, in den schwer zu behandelnden Subgruppen $61-77 \%$. Das Gesamtüberleben lag über alle Gruppen hinweg bei 18,4 Monaten (95\% CI 17,4-19,2). Dabei muss berücksichtigt werden, dass zirka drei Viertel der Patienten Sunitinib als Zweitlinientherapie nach Zytokinvortherapie erhielten [6]. Diese Ergebnisse haben eine hohe Relevanz in Bezug auf die manchmal schwierige Therapiesituation des klinischen Alltags (Abb. 1).

\section{Behandlungserfolg durch optimales Therapiemanagement}

Damit die Patienten einen größtmöglichen Nutzen von Sunitinib haben, sind drei Punkte besonders wichtig:

- Patienten sollten - ausgehend von der Standarddosierung von $50 \mathrm{mg}$ täglich im 4/2-Schema - eine optimale, individuell abgestimmte Dosis erhalten. Klinische Studien haben einen $\mathrm{Zu}-$ sammenhang zwischen Sunitinib-Exposition und Anti-TumorEffektivität (PFS und Gesamtüberleben) gezeigt [7].

- Die Sunitinib-Therapie sollte durch ein proaktives Nebenwirkungsmanagement begleitet werden.

- Die Therapie sollte bis zum gesicherten Krankheitsprogress fortgeführt werden, da sich gezeigt hat, dass die Ansprechrate im Therapieverlauf ansteigt (Abb. 2). Ebenso wurden mit zunehmender Dauer der Medikation Komplettremissionen beobachtet [1,2]. Ein optimales Therapiemanagement umfasst unter anderem Maßnahmen zur Prävention sowie zur Behandlung von Nebenwirkungen. Ziele sind dabei die Erhaltung der Lebensqualität und die Vermeidung unnötiger Schritte wie eine Unterbrechung bzw. Verzögerung der Therapie oder auch eine Dosisanpassung. Besonders wichtig ist dabei ein proaktives Vorgehen, das den Patienten in den Therapieprozess einbezieht. Dies beinhaltet eine umfassende Aufklärung, beispielsweise zu den zu erwartenden Nebenwirkungen und den beste-

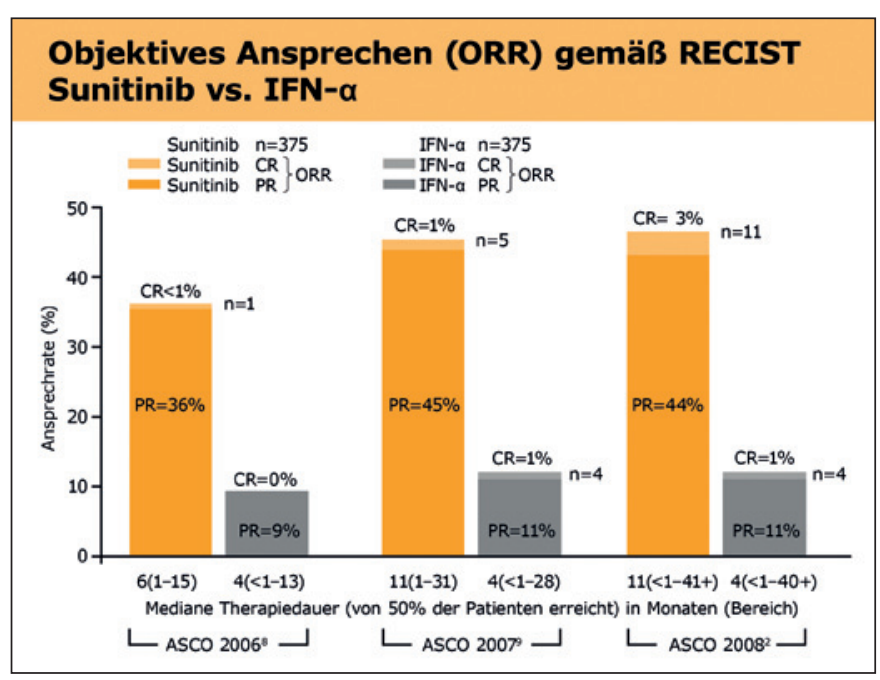

Abb. 2. Die objektive Ansprechrate unter Sunitinib erhöht sich im Therapieverlauf einschließlich der Anzahl kompletter Remissionen. 
henden Behandlungsmöglichkeiten. Wichtig ist auch, dass die Patienten den Zusammenhang zwischen Compliance und Therapieerfolg verstehen, um eine entsprechende Therapiedauer und damit einen maximalen Therapieerfolg zu gewährleisten.

\section{Hilfestellung für die Praxis: Broschüre «Sutent ${ }^{\circledR}$ Therapiemanagement»}

Um Behandler und Patienten im Therapieprozess möglichst umfassend zu unterstützen, wurden verschiedene Materialien entwickelt. So enthält die Broschüre «Sutent ${ }^{\circledR}$ Therapiemanagement» die verfügbaren Angaben aus der medizinisch-wissenschaftlichen Literatur (Abb. 3).

Abb. 3. In der Broschüre «Sutent ${ }^{\circledR}$ Therapiemanagement» wurden die verfügbaren Angaben aus der wissenschaftlichen Literatur zusammengetragen.
Zusätzlich bewerteten klinisch tätige Ärzte mit umfangreicher Erfahrung in der Anwendung von Sunitinib die publizierten Empfehlungen vor dem Hintergrund ihrer eigenen Erfahrung. Für Nierenkrebspatienten stehen unter anderem eine Informationsbroschüre sowie ein Patiententagebuch zur Verfügung.

\section{Fazit}

Sunitinib ist ein modernes und hocheffektives Medikament, das auf ein konsequent geplantes Studienprogramm sowie langjährige klinische Erfahrung verweisen kann und in der Erstlinientherapie des mRCC bevorzugt eingesetzt wird [10]. Das internationale Sunitinib-EAP für mRCC-Patienten gehört zu den größten bisher beim RCC durchgeführten klinischen Studien. Zusammen mit der Zulassungsstudie bietet es eine breite Evidenzbasis für den Einsatz des Multi-Kinase-Inhibitors in allen Patientenpopulationen. So traten keine neuen oder kumulativen Toxizitäten auf. Die prognostisch ungünstigen Subgruppen unterschieden sich bezüglich der Verträglichkeit von Sunitinib nicht von der Gesamtpopulation. Auch in der manchmal schwierigen Therapiesituation des Praxisalltags, in welcher Patienten oft mit zahlreichen Komorbiditäten belastet sind, konnte eine vergleichbare Wirksamkeit wie in der Phase-III-Studie erreicht werden. Die Therapie mit Sunitinib ist ambulant gut durchführbar und kann als ein Meilenstein beim mRCC bezeichnet werden.

\section{Disclosure Statement}

The author participated as investigator in the Sunitinib expanded access program.

\section{Literatur}

1 Motzer RJ, Michaelson MD, Hutson TE, et al: $\mathrm{Su}-$ nitinib versus interferon alfa in metastatic renalcell carcinoma (mRCC): updated efficacy and safety results and further analysis of prognostic factors. Eur J Cancer 2007;5(4S):301s, abstr 4509.

2 Figlin RA Hutson TE, Tomczak P, et al: Overall survival with sunitinib versus IFN-alpha as firstline treatment of mRCC. J Clin Oncol 2008;26 (15SI):256s, abstr 5024.

3 Shephard DR, Rini I, Garcia JA, et al: A multicenter prospective trial of sorafenib in patients (pts) with metastatic clear cell renal cell carcinoma (mccRCC) refractory to prior sunitinib or bevacizumab. J Clin Oncol 2008;26(suppl):abstr 5123.
4 Rini B, Hutson TE, Elson P: A prospective trial of Sorafenib in patients with metastatic clear cell carcinoma (mccRCC) refractory to prior sunitinib or bevacizumab ASCO GU 2008; \#346.

5 Tannir N, Wong Y, Kollmannsberger C, et al: Phase II trial of ABT-869 in advanced renal cell cancer (RCC) after sunitinib failure: efficacy and safety results. J Clin Oncol 2009;27:15s(suppl), abstr 5036.

6 Gore ME, Szczylik C, Porta C, et al: Safety and efficacy of sunitinib for metastatic renal-cell carcinoma: an expanded-access trial. Lancet Oncol 2009;10:757-763.

7 Houk BE, Bello CL, Michaelson MD, et al. Exposure-response of sunitinib in metastatic renal cell carcinoma (mRCC): A population pharmacokinetic/ pharmacodynamic (PKPD) approach. ASCO 2007; abstr 5027.
Motzer RJ, Hutson TE, Tomczak P, et al: Phase III randomized trial of sunitinib malate (SU11248) versus interferon-alfa (IFN- $\alpha$ ) as first-line systemic therapy for patients with metastatic renal cell carcinoma (mRCC). J Clin Oncol 2006;24(18SII):930s, LBA3.

9 Motzer RJ, Figlin RA, Hutson TE, et al: Sunitinib versus interferon-alfa (IFN- $\alpha$ ) as first-line treatment of metastatic renal cell carcinoma (mRCC): Updated results and analysis of prognostic factors. J Clin Oncol 2007;25(18S):241s, abstr 5024

10 Müller L, Münch A, Blumenstengel K, et al: Treatment of patients with advanced or metastatic renal cell carcinoma (RCC registry): a 'real life' treatment overview of non-trial patients in outpatient cancer centres in Germany. DGO 2009, abstr V861. 